\title{
COMPARAISON DE DIVERS MODES DE RATIONNEMENT DE LA POULETTE ET DE LEURS EFFETS SUR LES PERFORMANCES DE PONTE
}

\author{
J-C. BLUM, B. LECLERCQ et I. GUILLAUME \\ Station de Recherches avicoles, \\ Centre de Recherches de Tours, I. N.R. A., \\ Nouzilly $B 1$ \\ 37380 Monnaie (France)
}

\section{RÉSUMÉ}

Jour étudier l'influence du rationnement pendant la période de croissance, on constitue six lots de I 80 poulettes reproductrices parentales (souche de type ponte). Le lot témoin consomme toujours ad libitum des aliments complets. Deux lots disposent des mêmes aljments, ad libitum d'abord, puis en quantité également limitée après l'âge de huit semaines; pour l'un de ces lots la distribution est quotidienne (lot 2), pour l'autre, elle a lieu I jour sur 2 (lot 3). Les trois autres lots sont nourris comme les poulettes témoins après l'âge de 8 semaines, mais auparavant, depuis l'éclosion, elles subissent diverses privations : pour deux des lots, le régime consommé ad libitum est déficient soit à la fois en lysine et d'autres acides aminés (lot 4 ), soit en lysine seulement (lot 5) ; le dernier lot dispose quotidiennement d'une quantité limitée de régime complet (lot 6).

Le rationnement effectué à partir du régime équilibré, qu'il soit imposé dès l'éclosion (lot 6) ou à partir de 8 semaines (lots 2 et 3), permet une économie appréciable d'aliment et assure la meilleure efficacité alimentaire. Les retards de maturité sexuelle sont peu importants, mais ils font apparaitre une différence significative entre les lots 2 et 3 montrant l'importance du rythme d'alimentation chez les animaux rationnés : seul le rythme i jour sur 2 retarde la maturité sexuelle.

Pendant la période de ponte ( 280 jours), un même régime est distribué ad libitum à toutes les poules. Le nombre d'œufs pondus est souvent augmenté dans les lots antérieurement rationnés (lot 3 excepté), mais aucune différence n'est significative. Dans le lot 5, qui était exclusivement carencé en lysine, le poids moyen de l'œuf est systématiquement diminué alors qu'il demeure normal dans le lot 4 qui manquait à la fois de lysine et d'autres acides aminés. Le rationnement quantitatif précoce (lot 6) a une influence très originale : il assure d'abord un poids d'œuf normal mais ralentit l'augmentation en cours de ponte ; à partir du $3^{\mathrm{e}}$ mois, les cufs sont significativement plus petits que dans le lot témoin.

En définitive, le rationnement, pendant la croissance, paraît toujours bénéfique. Il devrait de préférence être institué dès l'éclosion; il est alors particulièrement bien supporté, conđuisant à une mortalité réduite, à une production d'œufs élevée et d'un poids le plus souvent satisfaisant. 


\section{INTRODUCTION}

On sait maintenant qu'il est possible de réduire les dépenses alimentaires pendant la période d'élevage sans nuire aux performances de ponte. Dans certaines souches et leurs croisements, on évite ainsi un engraissement préjudiciable. Les méthodes utilisées, leurs croisement avantages et leurs inconvénients, ont fait l'objet d'une revue bibliographique récente (LEE et al., I97I). Dans le rationnement quantitatif, 1'aliment complet est distribué en quantité limitée (volume déterminé ou réduction du temps d'accès aux mangeoires). Dans le « rationnement qualitatif », l'aliment est déséquilibré par un apport insuffisant ou excessif de certains nutriments dans le but de rédtuire l'appétit des animaux. Souvent, les rationnements débutent tardivement après plusieurs semaines de croissance maximum. Seules des méthodes qualitatives ont été utilisées dès l'éclosion : déficience en lysine seule (SINGSEN et al.,Ig64; SHERwood et al., Ig69) déficience en protéines (Summers, PEPPER et Moran, I969; LECLERCQ et al., I97o). Ainsi que LEE et al., (I97I) le soulignent, les diverses méthodes de rationnement ont été rarement comparées au cours d'un même essai. C'est ce type de comparaison que nous avons voulu réaliser en recherchant l'influence du mode de rationnement appliqué précocement (o à 8 semaines) ou tardir ament ( 8 à 20 semaines) pendant la croissance.

\section{MATÉRIEL E'T MÉTHODES}

\section{1. - Période de croissance}

Les poulettes appartiennent à une souche parentale de type Rhode Island Red (M 99, I. N. R. A., France). Séparées des mâles dès l'éclosion, elles sont élevées au sol dans un poulailler sans fenêtre, divisé en parquets de $3 \times 2$ mètres, contenant chacun 40 animaux. Des tubes fluorescents diffusent une lumière rouge de faible intensité. La période d'éclairement journalier de 24 heures les deux premiers jours est progressivement réduite jusqu'à une durée constante de 12 heures à partir de l'âge de 6 semaines.

Trois régimes isoénergétiques sont utilisés pendant la croissance; leur composition est indiquée dans le tableau r. Les teneurs en protéines et en lysine ont été exactement déterminées par macrokjeidahl pour les premières et par analyse chromatographique pour les secondes. Le régime 20 (20 p. IoO de protéines) est complet et équilibré pour des poussins de souche ponte pendant les premières semaines de vie; il ne diffère du régime $20-\mathrm{L}$ déficient en lysine que par la supplémentation en L-lysine. Le régime $\mathrm{I}_{4}$ est comparable au régime précédent par sa teneur en lysine $(0,63$ p. IOO); il en diffère par sa teneur en protéines (I4 au lieu de 20 p. IOO).

Le mode de distribution de ces trois régimes selon l'âge des animaux définit 6 lots de $24^{\circ}$ poulettes chacun (6 parquets, soit 6 répétitions par lot). Le protocole cxpérimental obéit au schéma suivant :

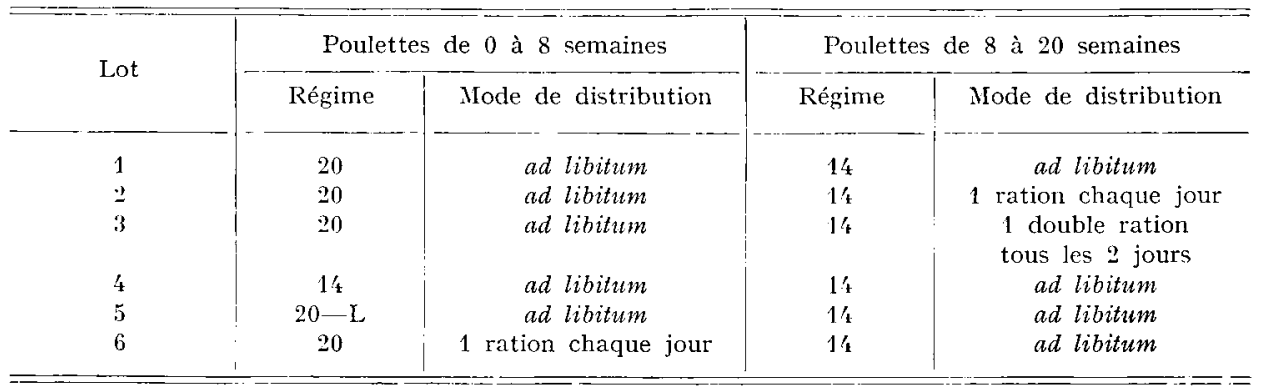


Le lot I (témoin) dispose de la meilleure alimentation pour un développement pondéral rapide selon un usage encore très répandu cn aviculture. Les lots 2 et 3 ont une croissance ralentie à partir de l'âge de 8 semaines par un rationnement quantitatif; la ration, fixée d'abord à $45 \mathrm{~g}$ par poulette et par jour, augmente progressivement jusqu'à 80 grammes à l'âge de 20 semaines. Dans le lot 2, la distribution de l'aliment a lieu une fois par jour. Dans le lot 3 les poulettes ne sont nourries qu'un jour sur deux; elles reçoivent alors une double ration et consomment donc la même quantité totale d'aliment. Dans les lots suivants, la croissance est ralentie dès l'éclosion par suite d'une déficience alimentairc (lot 4 : en divers acides aminés; lot 5 : en lysine seule) ou d'un rationnement quantitatif quotidien (lot 6 ). Ce dernier rationnement est adapté pour que la croissance des poulettes soit comparable dans le lot 6 d'une part et dans les lots 4 et 5 d'autre part.

TABLEAU I

Composition des régimes

\begin{tabular}{|c|c|c|c|c|}
\hline \multirow[b]{3}{*}{ Mais } & \multicolumn{3}{|c|}{ Période de croissance } & \multirow{2}{*}{ Période de ponte } \\
\hline & Réginle 20 & Régine 1't & Régine 20--L & \\
\hline & 60 & 27 & 60 & 40 \\
\hline Blé $\ldots \ldots \ldots \ldots \ldots \ldots \ldots \ldots$ & - & 30 & 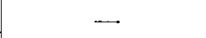 & 20 \\
\hline Avoine...$\ldots \ldots \ldots \ldots$ & 10 & 28 & 10 & 10,5 \\
\hline Tourteau de soja $4 t^{\prime} \ldots \ldots \ldots$ & - & - & - & 5 \\
\hline Tourteau d'arachide ......... & 15 & 4 & 15 & $-\cdots$ \\
\hline Tourteau de tournesol ......... & - & - & - & 10 \\
\hline Farine de poisson $70 \ldots \ldots \ldots$ & 0,5 & 3,5 & 0,5 & 2 \\
\hline Gluten de mais ........... & 9,5 & - & 9,5 & -.. \\
\hline Farine de Iuzerne...$\ldots \ldots \ldots$ & - & 2,5 & - & $: 3$ \\
\hline Carbonate de calcium $\ldots \ldots \ldots$ & 1 & 1 & 1 & (i) \\
\hline Phosphate bicalcique...$\ldots \ldots$ & 2 & 2 & $\because$ & 2 \\
\hline Sel vétérinaire $\ldots \ldots \ldots \ldots \ldots$ & 0,5 & $0, \overline{5}$ & 0,5 & 0,1 \\
\hline Bicarbonate de sodium ....... & - . & - & - & 0,25 \\
\hline DL-méthionine $\ldots \ldots \ldots \ldots \ldots$ & 0,02 & 一 & 0,02 & - \\
\hline L-lysine $\ldots \ldots \ldots \ldots \ldots \ldots$ & $0, \geq ?$ & 0,08 & - & - \\
\hline Complément vitaminique $\left({ }^{1}\right) \ldots$ & 1 & 1 & 1 & 1 \\
\hline Complément minéral $\left({ }^{2}\right) \ldots$ & 0,5 & 0,5 & 0,5 & 0,15 \\
\hline Taux protidique (mesuré) & 20,7 & 14,3 & 20,4 & 15,5 \\
\hline Teneur en lysine (mesurce) & 0,97 & 0,68 & 0,68 & \\
\hline Énergic métabolisable $\mathrm{kcal} / \mathrm{kg}$ & & & & \\
\hline (calculée) $\ldots \ldots \ldots \ldots \ldots \ldots$ & 2830 & $28: 30$ & $\geq 830$ & 2755 \\
\hline
\end{tabular}

(1) Composition du complément vitaminique: Vit. A : $800000 \mathrm{UI}$; Vit. $\mathrm{D}_{3}$ : $100000 \mathrm{UI}$; riboflavine : $0,4 \mathrm{~g}$; pantothénate de calcium : $0,7 \mathrm{~g}$; nicotinanide : $1 \mathrm{~g}$; Vit. $\mathrm{E}: 5 \mathrm{~g}$; BHT : $10 \mathrm{~g}$; chlorure de choline : $40 \mathrm{~g}$; avoine broyée : q.s.p. $1000 \mathrm{~g}$.

(2) Composition du complémcnt minéral: Sulfate de cuivre : $7 \mathrm{~g}$; Sulfate de cobalt : $1 \mathrm{~g}$; Molybdate de sodium : $0,5 \mathrm{~g}$; Sulfate de zinc $: 150 \mathrm{~g}$; Iodure de potassium : 1,5 g; Sulfate de manganèse : $200 \mathrm{~g}$; Oxyde de magnésium : $100 \mathrm{~g}$; Carbonate de magnésium : $80 \mathrm{~g}$; Sulfate de fer : $75 \mathrm{~g}$; Carbonate de calcium : $335 \mathrm{~g}$.

\section{2. - Période de ponte}

A l'âge de 20 semaines, les animaux sont transférés en poulailler de ponte. Celui-ci comprend 24 cases de $15 \mathrm{~m}^{2}$. Chaque case comporte une partie sur litière et une partie sur fosse à déjection. Les animaux provenant des 6 répétitions de chaque lot pendant la croissance sont répartis dans 4 cases (4 répétitions) pour la période de ponte. Les performances réalisées dans chacune des répétitions sont calculées par périodes de 28 jours du début à la fin de la ponte. Le nombre d'œufs pondus et le nombre de poules présentes dans chaque case ( 45 au début de la ponte) sont 
consignés quotidiennement. La quantité d'aliment consommé est déterminée à la fin de chaque période. Les oufs sont pesés individuellement pendant deux jours au début de la $3^{\mathrm{e}}$ semaine de chaque période.

Pendant toute la durée de la ponte, les poules consomment ad libitum un seul et même aliment : sa composition figure dans le tableau I. L'éclairement est augmenté progressivement à partir de l'âge de 20 semaines à raison de 30 minutes par semaine jusqu'à un maximum de ${ }^{7} 7$ heures.

\section{RÉSULTATS}

\section{I. - Croissance des animaux}

Les poids moyens des animaux et leur consommation figurent dans le tableau 2. Durant les 8 premières semaines de vie, les lots se répartissent en deux groupes d'une part les lots I, 2 et 3 , d'autre part les lots 4,5 et 6 dont la croissance est très ralentie par rapport aux trois premiers. Il faut remarquer que la carence en lysine seule réduit plus profondément la croissance que la carence en protéines totales, bien que la teneur en lysine des deux régimes soit identique. Dans le lot 6 la quantité d'aliment complet distribuée était prévue pour assurer le même gain de poids que les régimes déficients (lots 4 et 5), mais le rationnement a été un peu excessif. Manquant de lysine, les poulettes des lots 4 et 5 en ont cependant consommé I 2 et II 2 grammes entre 0 et 8 semaines, celles du lot 6 , Io grammes seulement; l'écart plus grand encore pour les autres nutriments explique la différence de poids à l'âge de 8 semaines.

Entre 8 et 20 semaines d'âge, les trois lots ralentis dès la naissance tendent à rattraper le lot témoin. En revanche, le poids vif moyen est réduit dans les mêmes proportions par les 2 types de rationnement tardifs imposés aux lots 2 et 3 . Ce retard disparaît d'ailleurs pendant la ponte. A l'âge de 63 semaines, il ne subsiste aucune différence significative entre les 6 lots.

Les quantités d'aliments ingérées pendant les 20 semaines sont, elles aussi, très différentes. Les lots 2 et 3 sont ceux qui ont économisé le plus d'aliment. Ensuite vient le lot 6 pour lequel la consommation par animal demeure inférieure de I $\mathrm{kg}$ environ à celle du lot témoin. L'efficacité alimentaire est sensiblement identique dans ces trois lots rationnés quantitativement, que le rationnement intervienne précocement (lot 6) ou tardivement (lots 2 et 3 ). Dans les lots I, 4 et 5 , les consommations ne diffèrent pas significativement entre elles de 0 à 20 semaines. Les efficacités alimentaires sont également voisines et moins bonnes que dans les lots 2,3 et 6 .

\section{2. - Mortalité}

La mortalité enregistrée au cours de la période de croissance et de la période de ponte est rapportée dans le tableau 3. Bien qu'il soit impossible d'attribuer une signification statistique à ces résultats, il faut remarquer que les lots retardés précocement subissent les pertes les plus faibles aussi bien au cours de la croissance que pendant la ponte. On enregistre la plus forte mortalité dans le lot témoin.

\section{3. - Maturité sexuelle et performance de ponte}

L'âge moyen à $50 \mathrm{p}$. Ioo de ponte figure dans le tableau 2. Les différences entre lots ne sont pas très prononcées. Les lots 3 et 5 sont les plus retardés. Le lot 3 l'est significativement par rapport aux lots I et 2, bien qu'il ait ingéré les mêmes quantités 


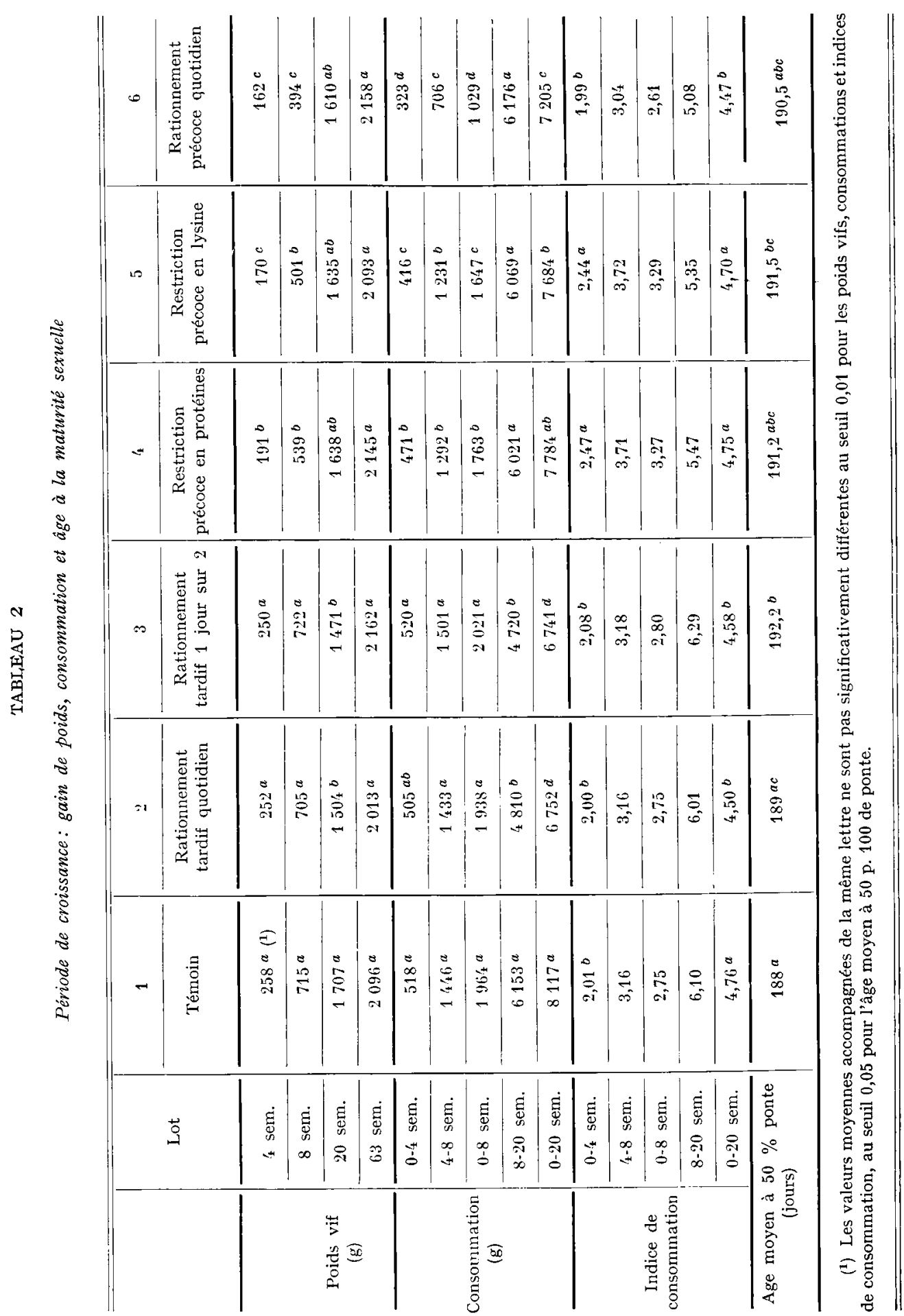


d'aliment que ce dernier, mais selon un rythme différent : double ration I jour sur 2 au lieu d'un repas quotidien entre 8 et 20 semaines d'âge. Par contre, aucune différence significative n'est mise en évidence entre les trois lots 4,5 et 6 dont la croissance avait été retardée de diverses manières pendant le jeune âge.

L'intensité de ponte, selon les périodes et selon les lots, fait l'objet du tableau 4.

TABLEAU 3

Mortalité (p. Ioo)

\begin{tabular}{|c|c|c|c|}
\hline Lots & $\begin{array}{c}\text { Période } \\
\text { d'élevage } \\
(0-20 \text { sem.) }\end{array}$ & $\begin{array}{c}\text { Période } \\
\text { de ponte } \\
(20-63 \text { sem. })\end{array}$ & $\begin{array}{c}\text { Mortalite } \\
\text { totale } \\
(0-63 \text { sem. })\end{array}$ \\
\hline 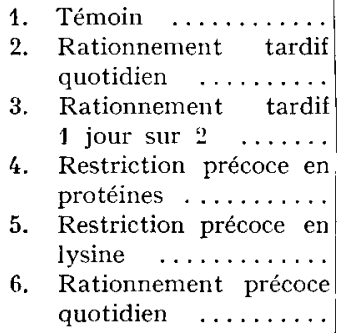 & $\begin{array}{l}3,7 \\
4,2\end{array}$ & $\begin{array}{r}19,7 \\
16,6 \\
15,7\end{array}$ & $\begin{array}{r}25,5 \\
20,3 \\
19,9\end{array}$ \\
\hline
\end{tabular}

TABLEAU 4

Évolution de l'intensité de ponte

et nombre d'œufs pondus en moyenne par poule à l'âge de 63 semaines

\begin{tabular}{|c|c|c|c|c|c|c|c|c|c|c|c|}
\hline \multirow{2}{*}{ Lots } & \multicolumn{10}{|c|}{$\begin{array}{c}\text { Intensité de ponte (Nombre d'œufs/100 poules/jour) } \\
\text { pendant les } 10 \text { périodes sucessives: }\end{array}$} & \multirow{2}{*}{$\begin{array}{c}\text { Nombre total } \\
\text { d'œufs } \\
\text { par poule** }\end{array}$} \\
\hline & $1 *$ & 2 & 3 & 4 & 5 & 6 & 7 & 8 & 9 & 10 & \\
\hline 1. Témoin $\ldots \ldots \ldots \ldots$ & 25,6 & 64,5 & 67,9 & 68,7 & 68,6 & 61,0 & 58,6 & 51,2 & 37,8 & 32,4 & 150 \\
\hline $\begin{array}{l}\text { 2. Rationnement tardif } \\
\text { quotidien } \ldots \ldots \ldots \ldots\end{array}$ & $18,{ }^{\prime}$ & 67,3 & 77,3 & 75,9 & 73,5 & $6\}_{t}^{\prime},{ }_{t}^{\prime}$ & 59,2 & $5 / 4,2$ & 46,4 & 32,0 & 159 \\
\hline 3. Rationnement tardif & & & & & & & & & & & \\
\hline $\begin{array}{l}1 \text { jour sur } 2 \ldots \ldots \ldots \\
\text { 4. Restriction précoce en }\end{array}$ & $11,{ }^{\prime}$ & 63,5 & 72,4 & 70,0 & 68,2 & 61,8 & 57,2 & 50,3 & 40,2 & 35,2 & 149 \\
\hline $\begin{array}{l}\text { protéines } \ldots \ldots \ldots \ldots \ldots \\
\text { 5. Restriction précoce en }\end{array}$ & 17,0 & 67,0 & 76,4 & 74,0 & 72,0 & 65,7 & 63,5 & 55,8 & 40,9 & 37,4 & 160 \\
\hline lysine $\ldots \ldots \ldots \ldots$ & 13,2 & 66,7 & 78,2 & 76,7 & 75,1 & 68,0 & 64,4 & 56,0 & 40,7 & 33,4 & 160 \\
\hline 6. Rationnement précoce & 153 & 633 & 74 & 746 & 711 & 646 & 619 & 596 & 41 & 345 & 154 \\
\hline & 10,0 & & $8, \mathrm{x}$ & 17,0 & 1,1 & $0.7,0$ & 2 & 02,0 & $71, \pm$ & $1 \times, 0$ & $10 x$ \\
\hline
\end{tabular}

* Chaque période dure 4 semaines. La période 1 commence à l'àge de 23 semaines. La période 10 se termine à l'àge de 63 semaines. Aucune différence n'est significative.

** $\frac{\text { Nombre total d'ceufs pondus }}{\text { Somme des nombres de poules relevés quotidiennement }} \times$ nombre de jours d'expérience. 
L'évolution de la ponte en fonction du temps est très semblable d'un lot à l'autre. Toutefois, la production est toujours supérieure dans les lots 4 et 5 restreints précocement en acides aminés (protéines ou lysine seule). En calculant le nombre moyen d'œufs pondus par poule présente, cette supériorité, bien que non significative, se traduit par un supplément de Io œufs par rapport aut lot témoin. Une même différence de Io œufs sépare les deux lots rationnés tardivement donnant l'avantage à la distribution quotidienne par rapport à l'alimentation I jour sur 2. Le rationnement précoce et quotidien (lot 6 ) permet une performance intermédiaire.

L'évolution du poids moyen de l'œuf est rapportée dans le tableau 5. Le poids augmente normalement pendant la ponte ; on doit cependant noter une particularité dans cette souche : un plafonnement et même une diminution les $9^{\mathrm{e}}$ et Io $^{\mathrm{e}}$ périodes. Les différences entre le lot témoin et les lots rationnés tardivement (2 et 3 ) sont faibles et rarement significatives. Il en est de même pour le lot 4 soumis à la restriction précoce en protéines, le poids de l'œuf n'y est diminué qu'au tout début de la ponte (première période). Par contre, les poulettes du lot 5 se singularisent en pondant des œufs systématiquement moins gros que celles des lots précédents; la réduction du poids moyen de l'ouf est significative dès le début et presque tout au long de la ponte. Une insuffisance d'apport portant exclusivement sur la lysine a donc une influence marquée en comparaison de la même privation imposée au lot 4 en même temps que d'autres déficiences en acides aminés.

TABLEAU 5

Poids moyen de l'auf pendant les dix périodès successives de 28 jours (en g)

\begin{tabular}{|c|c|c|c|c|c|c|c|c|c|c|c|}
\hline \multirow{2}{*}{ Lots } & \multicolumn{10}{|c|}{ Période } & \multirow{2}{*}{ Moy. } \\
\hline & 1 & 2 & 3 & 自 & 5 & 6 & 7 & 8 & 9 & 10 & \\
\hline 1 & $51,4 a *$ & $54,8^{a}$ & $55,7 a b$ & $58,5^{a}$ & $59,9^{a}$ & $60,5^{a}$ & $61,2 a b$ & $62,0^{a}$ & 61,5 & $60, t_{t}$ & 58,6 \\
\hline 2 & $50,8^{a b}$ & $5^{\prime}, 3^{b}$ & $55,2^{b}$ & $57,9 a b c$ & $60,6^{a}$ & $59,8 a b$ & $61,4 a b$ & $61,7 a b$ & 61,0 & 59,6 & 58,2 \\
\hline 3 & $51,2 a b$ & $55,1^{a}$ & $56,2 n$ & $57,7 b c$ & $59,8^{a}$ & $60,5^{a}$ & $62,0^{a}$ & $62,2^{a}$ & 62,8 & 60,7 & 58,8 \\
\hline 4 & $50,5^{b c}$ & $5 \frac{1}{4}, a b$ & $56,3^{a}$ & $58,3 a b$ & $60,1^{a}$ & $60,7^{a}$ & $61,0 a b$ & $61,7^{a b}$ & 61,9 & 59,9 & 58,5 \\
\hline 5 & $50,0^{c}$ & $53,7^{c}$ & $54,6^{b}$ & $57,2 c$ & $58,3^{b}$ & $58,9^{b}$ & $60,1^{b}$ & $60,8^{b c}$ & 60,5 & 59,1 & 57,3 \\
\hline 6 & $51,3 a b$ & $54,4 a b$ & $55,0^{b}$ & $56,6^{c}$ & $58,8^{b}$ & $59,3^{b}$ & $60,0^{b}$ & $60,5^{c}$ & 60,4 & 59,5 & 57,6 \\
\hline
\end{tabular}

* Les différences observées entre lots au cours de chaque période sont significatives lorsque les poids moyens ne sont pas suivis d'une même lettre.

Cependant l'action de la carence en lysine n'est pas réellement spécifique. Le poids de l'œuf est également diminué dans le lot 6 qui a subi un rationnement sévère dans le jeune âge. La diminution est significative au cours de nombreuses périodes. En fait, l'évolution du poids de l'œuf pendant la ponte est différente dans les lots 5 et 6 . Dans le lot 5 elle est parallèle à celle observée dans le lot témoin, le poids demeurant toujours inférieur de plus de I gramme. Dans le lot 6 aucune différence avec le lot témoin n'est perceptible pendant les deux premières périodes; l'écart devient significatif à partir de la $4^{e}$ période; dès lors, et jusqu'à la fin de la ponte, les lots 5 et 6 produisent des cufs de poids comparables. 


\section{DISCUSSION ETT CONCLUSION}

Une fois de plus, il est possible d'affirmer que les privations subies pendant la période de croissance ne compromettent pas les performances de ponte. Ainsi, nous confirmons les travaux de nombreux auteurs (LEE et al., I97I) et nos propres résultats antérieurs (LECLERCQ et al., I970; LECLERCQ et BLUM, I972). La comparaison des méthodes mises en œuvre dans cet essai aboutit à des constatations plus originales.

L,e rationnement institué tardivement à l'âge de huit semaines est d'usage courant, mais l'influence du rythme d'alimentation n'a guère été envisagée jusqu'à maintenant. Cependant l'un d'entre nous avait suspecté cette influence au cours d'une précédente étude (GUIL,AUmE, I968). Nous démontrons ici qu'un même niveau de rationnement retarde ou non la maturité sexuelle selon que l'aliment est distribué un jour sur deux ou quotidiennement, ces deux modes de distribution assurant par ailleurs un même gain de poids et une efficacité alimentaire identique. En outre, on peut remarquer que la distribution un jour sur deux est le seul procédé de rationnement (précoce ou tardif) qui n'a pas augmenté le nombre d'œuls pondus. Certes, ce dernier résultat n'est pas significatif, mais l'influence bénéfique du rationnement sur la production a été constatée au cours d'expériences précédentes (LECLERCQ et al., I970; LECLERCQ et BLUM, I972). La différence de ro œufs par poule entre les lots 2 et 3 est assez importante pour être prise en considération ; l'absence de signification peut tenir à la méthode de mesure : le contrôle par répétition a l'avantage d'être simple, mais il est bien moins précis que les contrôles individuels précédemment mis en œuvre. En définitive, le rythme d'alimentation après 1'âge de 8 semaines semble influencer électivement la fonction de reproduction : contrairement à la distribution un jour sur 2, le rationnement quotidien ne retarde pas la maturité sexuelle, il tend à accroître le nombre d'œufs pondus.

Parmi les méthodes utilisées pour ralentir la croissance dès l'éclosion, seule la carence en lysine (lot 5 ) est d'un usage courant. Nous constatons après plusieurs auteurs (Trammel, Greger et Couch, I968; Bougon et Meviis, Ig68) qu'elle induit une diminution significative du poids de l'œuf. Sans aucun doute la déficience d'apport intervient différemment selon la teneur en protéines du régime. Dans le régime I4 tous les acides aminés font défaut, la lysine étant probablement le facteur limitant primaire $(4,75 \mathrm{p}$. Ioo des protéines) qui a déterminé l'intensité de croissance chez les poulettes du lot 4 . Le régime $20-\mathrm{L}$, ne manque ni plus, ni moins, de lysine, mais le déficit est aggravé par la présence en quantité excessive des autres acides aminés ; ce déséquilibre a déprimé tout particulièrement la consommation et, par là même, le développement pondéral au cours de la première semaine de vie. Par la suite, les animaux du lot 5 ont eu une croissance comparable à ceux du lot 4 , mais contrairement à ces derniers, ils ont extériorisé la déficience en lysine par un symptôme caractéristique : présence de nombreuses plumes blanches à l'âge de 4 semaines. Il n'est donc pas surprenant de constater une différence d'action des deux régimes, pauvre en protéines ou en lysine seule sur les performances de ponte. Il faut cependant souligner que la réduction du poids de l'œuf ne résulte pas d'une façon absolument 
spécifique de la carence en lysine. En effet, nous avons déjà constaté que des régimes pauvres en protéines avaient parfois le même effet lorsqu'ils étaient distribués pendant les premières semaines de vie (LECIFRCQ et al., I970; LECLERCQ et BLUM, I972). Dans cet essai, un autre type de privation, le rationnement quantitatif institué dans le lot 6 , a un effet analogue quoiqu'apparaissant plus tardivement. Force est d'admettre que des conditions nutritionnelles particulièrement défavorables lors des premières semaines de vie modifient de façon irréversible le développement de certains organes ou de certaines fonctions de telle sorte que les compensations ultérieures ne seront jamais totales. Cette " hypotrophie "n'est pas en relation directe avec le développement pondéral : le rationnement quantitatif précoce ralentit plus la croissance et diminue moins le poids de l'œuf que la déficience en lysine ; par ailleurs la poule efface peu à peu le retard de croissance au cours de la ponte pour atteindre finalement un poids normal dans tous les lots. En fait, l'influence du rationnement varie selon la nature du déficit alimentaire imposé entre o et 6 semaines. L'équilibre entre constituants de la ration et les apports relatifs d'énergie, protéines, acides aminés ont peut-être plus d'importance que la réduction de croissance proprement dite. Les compensations qui surviennent tardivement font disparaitre les différences de poids, ce rattrapage étant totalement indépendant des performances de ponte.

D'un point de vue pratique, il faut souligner que toutes les méthodes de rationnement ou de restriction pendant la croissance sont bénéfiques. Elles permettent une économie d'aliment sans préjudice pour le nombre d'œufs pondus. Le retard de maturité sexuelle peut être recherché pour éliminer une partie des œufs défectueux du début de ponte; de toute façon, ce retard est toujours minime. Les méthodes tendant à ralentir la croissance dès l'éclosion présentent des avantages incontestables. D’une réalisation aisée, elles sont très bien supportées et améliorent même la vigueur des animaux : la réduction de la mortalité (ro à 15 p. IOO) est appréciable; son absence de signification statistique peut être liée au petit nombre des répétitions. Le nombre d'œufs pondus est plutôt augmenté. La diminution du poids de l'œuf est très peu importante, elle affecte seulement les lots qui ont subi une privation éprouvante pendant leur jeune âge. A cet égard, une carence globale en protéines paraît préférable à une déficience en la seule lysine. Le rationnement quantitatif quotidien d'un régime complet pourrait être choisi et appliqué dès 1'éclosion; il assure à 1'aliment une très bonne efficacité et constitue donc un procédé économique ; son influence sur l'évolution du poids de l'œuf est remarquable : normal au début, le poids n'est réduit que tardivement, ce qui peut constituer un avantage lorsque les poules pondent des œufs trop gros à la fin de la ponte.

Rę̧u pour publication en mai 1972.

\section{SUMMARY}

COMPARISON OF VARIOUS RESTRICTED FEEDING METHODS IN PUI IETS AND OF THEIR EFFECTS ON SUBSEQUENT LAYING PERFORMANCES

Six groups of 180 parental breeder pullets (laying type strain) were used to study the effect of restricted feeding during the growing period. The control group always received balanced diets ad libitum. Two groups were fed the same feeds, ad libitum first and then restricted after 
8 weeks of age. The diet was fed daily to one of these groups (group 2 ) and I day out of 2 to the other group (group 3). The three other groups were fed as the controls after 8 weeks of age, but prior to that, $i . e$. from hatching, they had been subjected to various restrictions : for two of the groups the diet fed ad libitum was deficient either both in lysine and other amino acids (group 4), or in lysine only (group 5). The last group received every day a limited amount of balanced feed (group 6).

Restricted feeding carried out with the balanced diet either from hatching (group 6) or from 8 weeks of age (group 2 and 3 ), allowed appreciable sparing of food and ensured the best feed efficiency. Delay in sexual maturity was not very great, but there was a significant difference between groups 2 and 3 showing the implications of the feeding rhythm in the restricted animals only the I day out of 2 rhythm caused delay in sexual maturity.

During the laying period (280 days), the same dict was fed ad libitum to all birds. The number of eggs laid was often higher in the previously restricted groups (except group 3), but there was no significant difference. In group 5, fed diets deficient in lysine only, the mean egg weight was always smaller, whereas it remained normal in group 4 fed dicts deficient both in lysine and in other amino acids. Early quantitative feed restrictions had a very unusual effect : it first gave normal egg weight, but then affected the egg weight increment during the laying period; from the 3 rd month, the eggs were significantly smaller than those of the controls.

It may be concluded that restricted feeding during the growing period seems to be always beneficial. It has to be applied from hatching, since it is particularly well accepted at that moment. So it leads to reduced mortality, higher laying rate and, generally, satisfactory egg weight.

\section{RÉFÉRENCES BIBIOGRAPHIQUES}

Bougon M., Mevel M., ig68. IEffets d'une carence en lysine d'un aliment distribué à différentes varićtés de pondeuses pendant les dix premières semaines d'élevage. Bull. Inf. Ploufragan, 48, r5-28.

Guillaume J., I968. Influence of daily restricted feeding and skip a day feeding on sexual maturity and body composition of pullets. Can. Poult. Rev., 92, 26-28.

LECLERCQ B., BLUM J.-C., I972. Effets de diverses restrictions alimentaires chez la poulette reproductrice chair dw sur les performances de ponte. Ann. Génét. Sél. anim., 4, 259-27o.

Leclerce B., Simon J., Blum J.-C., CAlet C., I970. Influence des restrictions alimentaires intervenant dès la naissance sur les performances de ponte de deux souches de poulettes. Ann. Zootech., 19, $333^{-346 .}$

Lee P. J. W., Gulliver A.-L., Morris T.-R., I97I. A quantitativc analysis of the literature concerning the restricted feeding of growing pullets. Brit. Poult. Sci., 12, 413-437.

Sherwood D.-H., Caskey C.-D., Jones J.-E., Smitir S.-B., Van Wormer M.-C., Eaton J.-B., r969. The use of lysine imbalanced feeds for starting or growing chicks. Poult. Sci., 48, I319-1328.

Singsen F. P., Naged. J., Patrick S. G., Matterson L. D., ig64. The effect of a lysine deficiency on body weight and age at sexual maturity of meat type pullets. Poult. Sci., 43, 786-787.

Summers J.-I)., Pepper W. F., Moran F. T., ig69. Use of amino acid imbalanced and low protein starting rations for the rearing of egg production type pullets and subsequent performance of these pullets when placed on laying rations of varying protein levels. Poult. Sci, 48, I35 I-I358.

Trammel J.-L., Greger C. R., Covch J. R., I968. Low lysine diets for rearing replacement pullets. Poult. Sci., 47, I 727 . 